\title{
New comprehensive surgical curriculum of pre-graduate surgical education
}

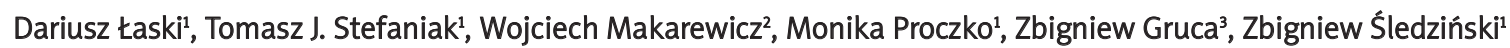 \\ 1Department of General, Endocrine and Transplant Surgery, Medical University of Gdansk, Poland \\ 2Department of Oncologic Surgery, Medical University of Gdansk, Poland \\ 3Pomeranian Foundation for Progress in Surgery, Poland
}

Videosurgery Miniinv 2013; 8 (3): 200-210

DOI: 10.5114/wiitm.2011.33756

\begin{abstract}
Introduction: Surgical education has become one of the most important directions in modern surgery evolution. To meet growing need for appropriate training in laparoscopic and, even more importantly, classic surgical skills, a curriculum involving contemporary tuition methods is needed. Advanced, structuralised training, which includes advanced technologies like virtual reality training, video coaching and motivative aspects of competition, seems to be important for an adequate education programme.

Material and methods: In academic years 2009/2010 and 2010/2011 the Department of General, Endocrine and Transplant Surgery of the Medical University of Gdansk together with the Pomeranian Foundation for Progress in Surgery organized $4480 \mathrm{~h}$ of training in that area of classic (2744) and laparoscopic (1736) skills. Both groups were involved in the programme of training in which the two most important aspects were reliable evaluation of the results and effective motivation to work. Skill evaluation at different stages of the programme were based on completion time and quality measurements. Apart from that, at the end of the course, the participants completed a questionnaire on their subjective perspective on this innovative curriculum, the quality and stability of the skills they obtained.

Results: In both arms of the programme (laparoscopic and classic) a statistically significant improvement was obtained as early as after the second and third sessions in half of the exercises. The acquired skills were stable over time, as proved by the plateau of completion time achieved in 11 out of 12 exercises. The results of the post-training questionnaire revealed that the participants were very satisfied with the structuralised form of training and appreciated the motivational role of competition.

Conclusions: Contemporary surgical training should be organized as a systematic, well-evaluated and goal-oriented programme similar to the one proposed by our team. The use of contemporary training aids should be utilized in training of every surgical skill, not only laparoscopy. This form of training, associated with the component of competition, enables good and stable results to be achieved, as well as high satisfaction of trainees.
\end{abstract}

Key words: surgical education, virtual laparoscopy, virtual trainers, box trainers, classic techniques training, surgical curriculum, skills laboratory, mentoring, video coaching.

\section{Introduction}

The current model of surgical education in Poland does not contain precise recommendations about the role of manual training in the laboratory, which is becoming increasingly popular in the USA (FLS programme) [1-6] and in Western Europe (LLS) [7], espe-

Address for correspondence:

Tomasz J. Stefaniak MD, PhD (surgery), PhD (health psychology), MBA, Department of General, Endocrine and Transplant Surgery,

Medical University of Gdansk, 14 Smoluchowskiego St, Gdansk, Poland, phone: +48 5834938 10, e-mail: wujstef@gumed.edu.pl 
cially regarding laparoscopic training. In January 2011 the European Consensus on learning basic laparoscopic skills with virtual trainers was published [8]. That Consensus outlines the training requirements resulting from the reflections of 7 leading centres in Europe, but refers to the methodology of teaching (virtual training), which firstly is virtually unavailable in Poland, and secondly, by many authors is considered to be insufficiently technically refined. Furthermore, these programmes rarely exist at the level of pre-diploma education. Both in Poland and abroad, there are no structured courses for students, either in terms of manual laparoscopic training, or in much more necessary and reasonable basic manual skills training in classical surgery. In Poland, the first contact with laparoscopy for the young resident or houseman is usually just in the operating room. The use of laboratory training enables simplification of the learning curve, and thus increases task orientation, effectiveness and efficiency of the young surgery adept and the safety of operation as a consequence. Of course, surgery is more than just manual skills. To carry out a safe operation, one requires substantive knowledge of anatomy, physiology, pathology and knowledge of the surgical technique, but these have a strong position in the pre- and postgraduate training. Enabling a young adept laboratory training of basic manual skills produces a reflex action. The acquired skills and movement patterns do not require later excessive concentration of the adept, which enables a greater focus on other aspects of safe operation. Regarding training in classical techniques - such as suturing using tools, or tying by hand - it formally exists in the curriculum of students, but there have not yet been developed standards or a uniform formula of such training. It is, apparently, extremely desirable in the context of the use of basic surgical skills by representatives of a number of specialties, some of which will not have the opportunity to participate in the full training cycle reserved for surgery house physicians.

\section{Aim}

The aim of this study was to evaluate the objective effectiveness (to improve the results of the standardized tasks) and subjective effectiveness (opinion of trainees) of the training programme within the scope of manual skills of classical and laparoscopic surgery developed by a team of the Clinic of General,
Endocrine and Transplantation Surgery, Medical University of Gdansk and the Pomerania Foundation of Surgery Development.

\section{Material and methods \\ Teaching Programme}

The Surgical Curriculum consisted of two main parts: the so-called laparoscopic arm (LapSkills), practising on physical trainers with elements of training on virtual trainers; and the traditional techniques arm (ClassSkills), which provides practical learning of surgical suturing and binding.

The training time consisted of $28 \mathrm{~h}$ divided into 7 sessions of $4 \mathrm{~h}$ each. Between sessions occurred one month intervals.

Recruitment for elective classes was carried out by means of enrolment of students interested in surgery (elective). Students could sign up for both courses separately and the two combined.

\section{Laparoscopic training (LapSkills)}

The laparoscopic course in the academic year $2009 / 2010$ was attended by 27 students, and in 2010/2011 35, an average of 4 patients (2-5). The training programme for each group was the same. After completing the initial survey students were acquainted with the principles of safe use of the trainers and then they were trained on a virtual laparoscopic trainer. In the next stage, they had an opportunity to practise on the physical trainer (box trainer) preceded by instruction and practical tips along with a presentation of how to use the laparoscopic camera (the ability to focus the image, set the image in the horizontal position, basic navigation of the camera and proper centring of the image on the OS). Each time before the introduction of the new exercises, a theoretical instruction took place and the presentation of the proper implementation of an exercise with demonstration of common mistakes and how to avoid them. Participants then performed a trial exercise familiarizing themselves with the new job, but not more than 3 times. During this phase, participants were able to ask instructors questions and get technical advice with repetitive error correction.

Participants had at their disposal 2 identical laparoscopic training sets made by Carl-Storz. After each repetition of the exercise, students divided into 


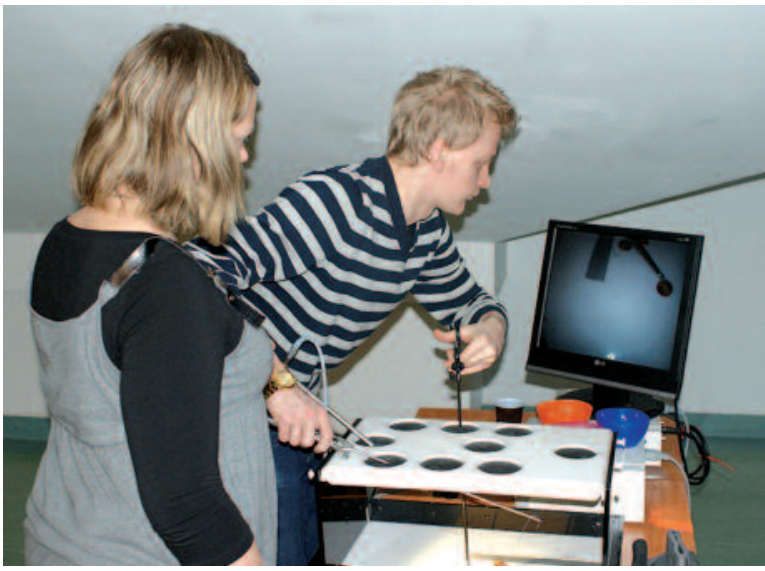

Photo 1. Exercises on a box trainer - Education Center of Pomeranian Foundation for Progress in Surgery 2009/2010

pairs changed roles. One acted as the operator, and the second as assistant camera operator.

On result cards, where personal information had been encoded, the instructors marked the time of the exercise. In case of a critical error the instructor interrupted the exercise. The exercise had to be repeated. Three-times occurrence of any critical errors (the same or different errors) resulted in an appropriate remark on the results in the results card for the exercise. At the end of the measurement of time, the instructor gave practical advice and corrected technical errors of the participant.

Each session began with a single repetition of previously completed training, and then presented more advanced tasks. To increase the motivation of the participants, there was introduced an element of competition between the course participants and an opportunity to closely follow the progress of their training by displaying the results (ranking) on the website.

\section{Curriculum}

\section{Exercise 1 - "10 buttons in a bowl" (Photo 1)}

This exercise consisted in placing 10 of 11 buttons distributed throughout the simulator chamber in a bowl. Buttons, always the same set of buttons of different shape, were randomly distributed in the central part of the chamber, and a cup set at the opposite wall of the chamber to the trainee.

The task was performed interchangeably, firstly with the dominant hand, and then the non-dominant one. Laparoscopic tools were introduced in the first
2 ports and the camera in the first seat of the middle row. For the hand performing exercises a dissector was used, but in the "helper" hand, depending on the preference of the participant, another dissector or a simple grasper was used.

The following were considered as critical errors: release of tools placed inside the trainer, overturning of a bowl placed in a chamber or its substantial displacement, placing in the bowl more or less than 10 buttons, hitting the wall of the trainer with one of the tools.

Aim of the exercise: Learning basic navigation in laparoscopy, communication with the cameraman, precise holding, for the person operating the camera the ability to direct the camera at the selected object, maintaining the proper horizon.

\section{Exercise 2 - " 5 buttons into a bowl - the inverted camera"}

This exercise was to put 5 of 6 spaced buttons in the trainer chamber into a bowl with inverted optics. The task was performed interchangeably entirely first by the dominant hand, then the non-dominant one, as in the first exercise. Critical errors were as in exercise 1.

Aim of the exercise: Learning basic navigation in reverse optics - camera opposite the operator, communication with the cameraman, precise holding, for the person operating the camera the ability to direct the camera at the selected object, maintaining the proper horizon.

\section{Exercise 3 - "Threading the buttons $\times 5$ "}

This exercise involved passing a needle through a hole in a button held in the left hand, the manoeuvre to be repeated five times. To this end a simple needle holder was used, selected for the dominant hand. For the non-dominant hand a simple preparator or grasper could be used, depending on the preference of the practitioner. The needle was to be held with clockwise rotation - so-called forehand. The button (with four holes) could be held in two ways, grasping the edge of the button or putting the jaws of the preparator inside the holes and closing the tool. The needle had to be threaded through the raised button, then intercepted and a suture threaded through the hole. Practice was continued until five buttons were threaded.

The following were considered as critical errors: dropping the tools inside the trainer and hitting the 
wall with the edge of needle or threading the needle other than prescribed in this exercise.

Reprimands were given in case of dropping a needle or a button.

Aim of exercise: Learning laparoscopic needle holder, the ability to lift the needle and proper positioning in the tool, the ability to capture a needle, precise manipulation of two tools, coordination of movements of both tools.

\section{Exercise 4- "Threading the buttons with backhand x3"}

This exercise involved putting a needle through the button hole in the needle in inverted orientation, so-called backhand, the manoeuvre to be repeated three times. Exercise and critical errors were the same as in exercise 3.

Aim of exercise: Learning laparoscopic needle holder, the ability to lift the needle and proper positioning in the tool, the ability to capture a needle, ability to work with a tool in backhand, precise manipulation of two tools, coordination of movements of both tools.

\section{Exercise 5 - "Tying a knot on the cup handle (3 knots)"}

This exercise involved tying three surgical knots (flat) single on the cup handle placed in the trainer chamber. The task was performed by using two simple laparoscopic needle holders. After tightening the knot the sequence was repeated twice more.

The following were considered as critical errors: dropping the tools inside the trainer chamber, hitting the walls of the simulator with a tool, overturning a cup or breaking a garter, tying knots in a way other than that recommended by the instructor and insufficient tightening of knots.

Purpose of exercise: Learning to use a laparoscopic needle holder, the ability to suture intracorporeal surgical knots, ability of soft and spot putting force of laparoscopic tools, e.g. by tightening knots, manipulating two tools, coordination of movements of both tools.

\section{Virtual laparoscopic trainer}

An additional element of the training comprised tasks carried out in virtual reality on LapVR trainers (Immersion Medical, USA) (Photo 2) After brief training and demonstration of exercises students performed once each of the three available exercises on the trainer. Such sessions were repeated at the first, fourth and seventh (last) meeting. Exercises on a virtual trainer were also a measuring tool allowing for more advanced training measures (such as the path length of the dominant and non-dominant hand). For trainees there were available 3 of 4 jobs in the "Basic skills" category. The difficulty level was set to "Basic".

\section{Exercise 1 - "Peg Transfer" - moving the capsules}

The student's task was to move without damage (at this level of difficulty) four elongated capsules and place them on a special stand with deep, narrow holes, alternately using the right and left hand. During an exercise, the trainer made a series of automatic measurements of quality parameters - including motion path length, time, number of errors for each hand, etc.

Aim of the exercise: Learning basic navigation in laparoscopy, precise holding, moving an object without crushing, learning of rotation around the long axis of the tool and tool axis coordination, the axis of the held object towards the target point.

\section{Exercise 2 - "Cutting skill" - cutting fabric in a virtual space}

The task was to cut out a circle of fabric stretched over a two-dimensional space that reflects three-

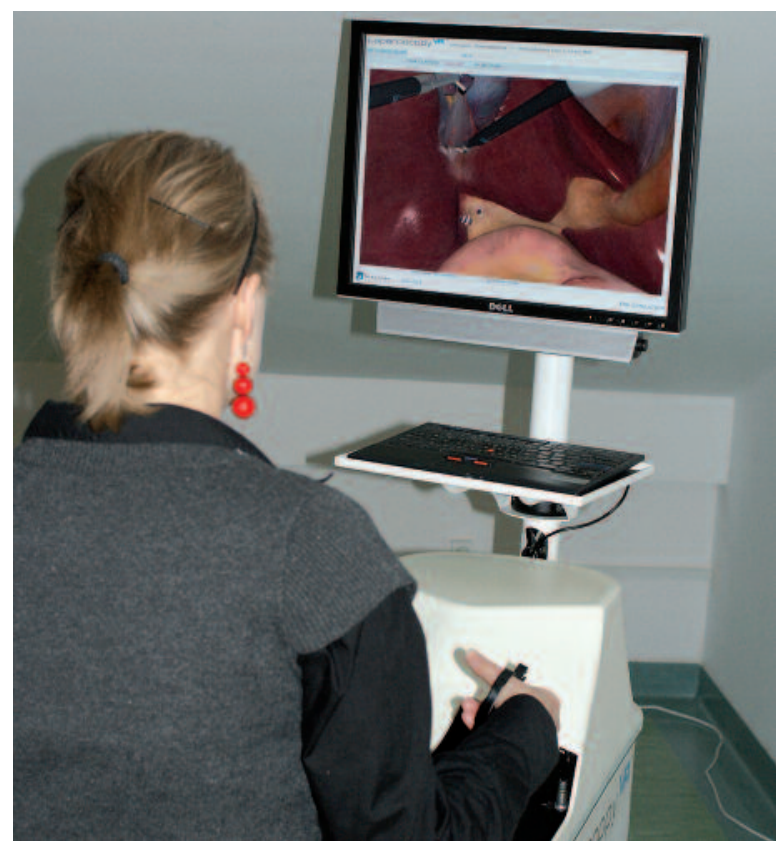

Photo 2. Exercises on virtual trainer - Education Center of Pomeranian Foundation for Progress in Surgery 2009/2010 


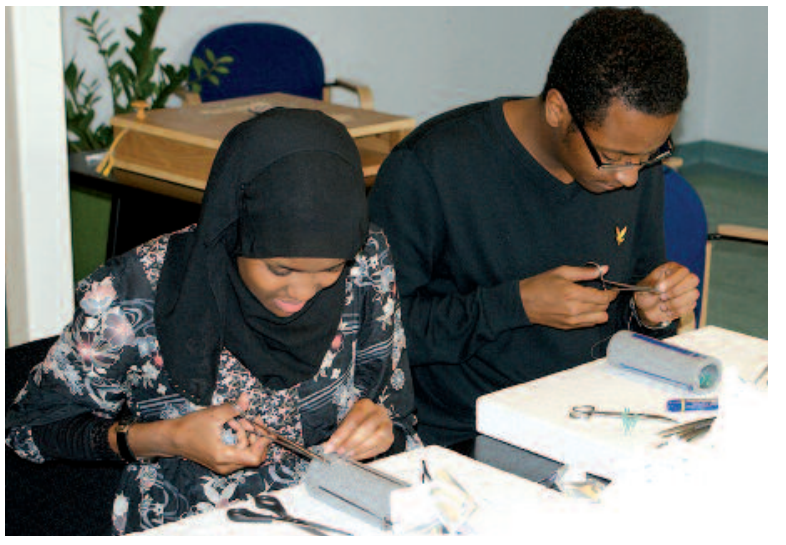

Photo 3. "ClassSkills" - tools suturing techniques - Education Center of Pomeranian Foundation for Progress in Surgery 2009/2010

dimensional space. This exercise allows one to assess the effectiveness of reflecting the depth of field and a sense of distance without a clear point of reference. In addition to the motion path and time for the task, it included measurement of the pressure on the fabric and cutting accuracy.

Aim of exercise: Learning to use laparoscopic scissors, precise cutting according to planned pattern depth evaluation in a 3D to $2 D$ conversion, learning application of voltage to the tissues to facilitate cutting and dissection.

\section{Exercise 3 - "Clipping skill"}

It is the task of clipping a blood vessel in specific places and cutting the vessel at the end of the procedure. In this task, apart from the standard parameters, the accuracy of placing clips and precision of cutting, the number of successfully and unsuccessfully placed clips, and the potential loss of blood in case of clipping leaks are recorded.

It can be concluded that the virtual trainer, despite not ideally reflecting the reality in the context of the image, allows for a careful analysis of the psycho-motor performance of the test-taker, which is very useful to monitor the progress of the student performing another task on a classic trainer, which has a better reflection, but a smaller range of measurement parameters.

\section{Classical techniques training}

The classical techniques training in the academic year 2009/2010 was attended by 44 students, and in the year 2010/2011 by 54, an average of 5 patients.
The training programme for each group was the same. After completing the initial form containing demographic data and a series of questions defining the profile of interests, and subjective evaluation of their manual skills, the students moved on to exercises. After a short briefing and presentation of proper implementation of exercises with a demonstration of the common mistakes and how to avoid them, the students performed some initial iterations, and then with an instructor performed the task on time.

Training sessions included alternating tasks using tools suturing techniques (Photo 3) and manual techniques. At each session completed exercises were repeated once and a new exercise of higher difficulty level was introduced.

Quality control was as in the case of laparoscopic techniques. In order to motivate students, like in the laparoscopic arm, the ranking of results of individual exercises was placed and updated on the web.

Participants had at their disposal a set of surgical tools, a constructed proprietary tool to suture knots in the body depth (Photo 4) and suture knots under tension, and proprietary kit to put on the ligatures (Photo 5).

\section{Exercise 1- 10 single simple knotted sutures}

The student's task was to make 10 single knotted sutures on a polyurethane sponge. Each had to be made according to the recommended criteria - the number of knots 10 , symmetrical, the distance between the sutures in the range of $8 \mathrm{~mm}$ to $10 \mathrm{~mm}$. The critical errors were blatant violations of quality criteria or plastic imitation of tissue damage.

Aim of exercise: Learning to use and hold the needle holder and surgical tweezers, grasping and learning the proper positioning of the needle and operating the needle in the tissue in accordance with the surgical technique, the ability to correctly tie surgical knots, learning precise surgical suturing, high reproducibility of the task.

\section{Exercise 2-25 knots tied by hand at the depth of the tool (Photo 4)}

In this exercise, students had to tie tight 25 knots using one of the demonstrated hand techniques using both hands alternately. In addition, the knots had to be brought into the chest depth to simulate ligature structure located deeper inside the operated area. The break of suturing or too loose knots was considered as a fatal error. 


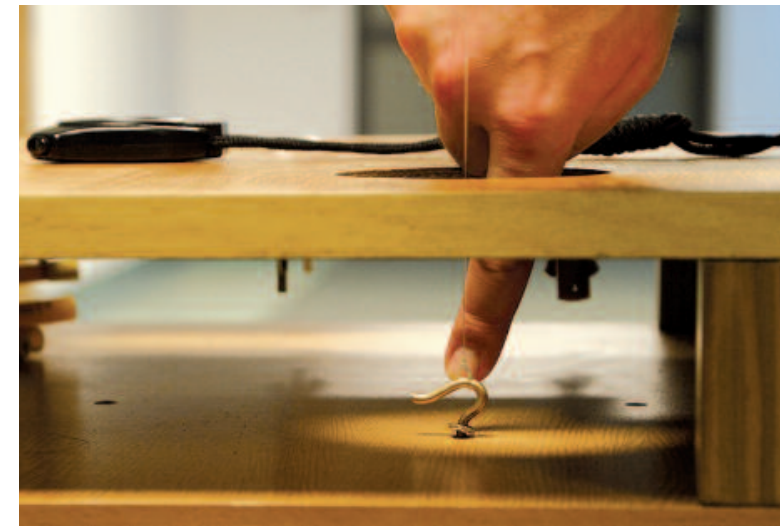

Photo 4. "ClassSkills" - hand suturing techniques - task 2 - Education Center of Pomeranian Foundation for Progress in Surgery 2009/2010

Aim of the exercise: Learning the correct tying of surgical knots for both dominant and non-dominant hand, the ability to put the knot into the depths and its accurate tightness.

\section{Exercise 3-10 vertical mattress stitches}

The student's task was to make 10 single vertical mattress stitches on a polyurethane sponge. Quality and critical errors were as in the first exercise.

Aim of exercise: Learning to use and to properly hold the needle holder and surgical tweezers, learning the proper positioning of the needle and operating the needle in the tissue in accordance with the surgical technique, learning the ability of correct stitching of surgical sutures, learning precision and high reproducibility of the task.

\section{Exercise 4- 5 ligature on the fabric (Photo 5)}

In the exercise, participants had to tie five ligatures, so-called "in loco", made of five stitches each, on a round piece of leather, as a simulation of a small blood vessel. A fatal error was breaking of suturing, moving the fabric outside the designated area or its excessive lifting. In addition, the knots had to be stitched tight.

Aim of the exercise: Learning of precise and delicate setting of surgical ligatures, without excessive movement and stretching of stitched structure.

\section{Exercise 5 - continuous suture, length of $10 \mathrm{~cm}$}

The student's task was to make a 10-cm simple continuous suture on a polyurethane sponge. Quality

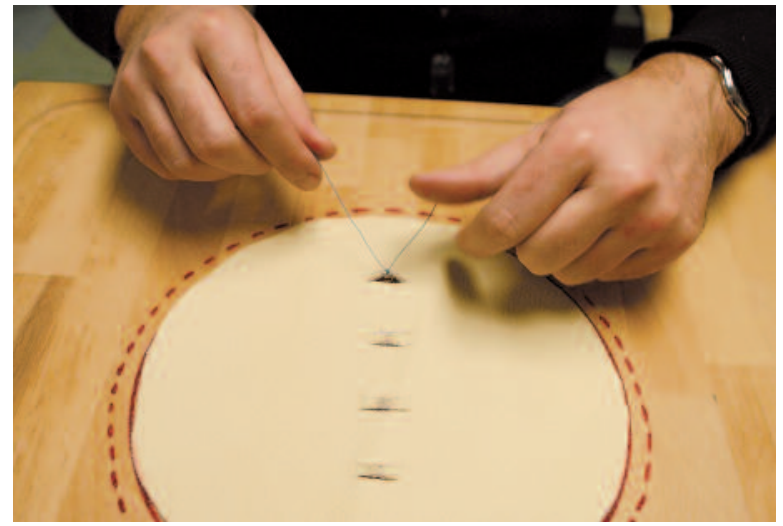

Photo 5. "ClassSkills" - hand suturing techniques - task 4 - Education Center of Pomeranian Foundation for Progress in Surgery 2009/2010

and critical errors were as in the first and third exercise.

Aim of exercise: Learning to use and to properly hold the needle holder and surgical tweezers, learning the proper positioning of the needle and operating the needle in the tissue in accordance with the surgical technique, learning the ability of correct stitching of surgical sutures, learning precision and high reproducibility of the task.

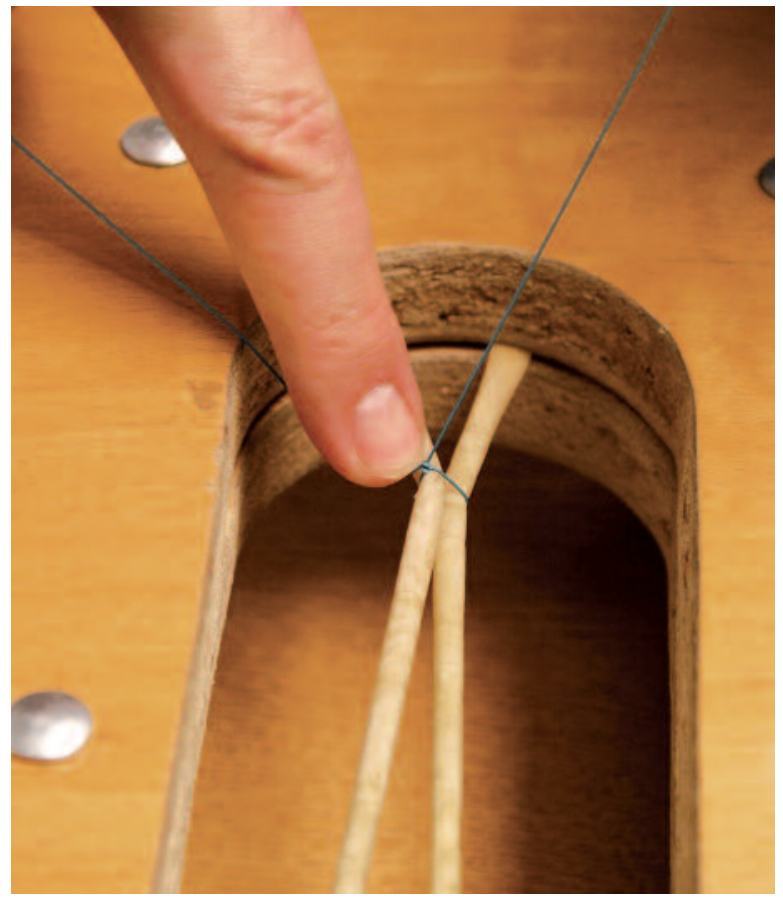

Photo 6. "ClassSkills" - hand suturing techniques - task 6 - Education Center of Pomeranian Foundation for Progress in Surgery 2009/2010 


\section{Exercise 6 - tying three ligatures under tension (Photo 6)}

In this exercise, the student was responsible for tying 3 ligatures under tension closing two taut Foley catheters as a simulation of the fascial layers bond. Critical errors were breaking of ligature and not full closing of catheters.

Goal of exercise: Learn how to make hand surgical knots under high tension of surrounding tissues

\section{Survey questionnaire}

All the trainees answered, before the first class, the questions of the questionnaire to determine their manual, psycho-motor and experience-related (video games, models, etc) suitability.

After the last session, participants completed a questionnaire exploring course satisfaction rate, subjective feelings about its effectiveness and attractiveness, and possible changes and improvements.

\section{Statistical analysis}

Most of the variables describing course evaluation were characterized by continuity and normal distribution, and therefore, in their measurement Student's $t$-test and ANOVA were used. Non-parametric variables were assessed via Mann-Whitney U-test. Descriptive variables of a discrete character were assessed by $\chi^{2}$ test. Each time, the level considered statistically significant was $p<0.05$. Analyses were conducted using
Statistica 9.0 PL software licensed to the Medical University of Gdansk.

\section{Results}

During the academic year 2009/2010 a total of 2212 man-hours of classes were carried out, including 980 in the laparoscopic arm (LapSkills) and 1238 in the classic arm (ClassSkills). In the academic year 2010/2011 a total of 2268 man-hours of classes were carried out, including 756 in the laparoscopic arm (LapSkills) and 1512 classical arm (ClassSkills). Data on the size, age and sex of the participants are shown in Table I.

Statistically significant improvement of the results was reported in the laparoscopic group after 2 repeats of exercise 1 (Scheffe post-hoc, $p<0.05), 2$ repeats of exercise 2 (Scheffe post-hoc, $p<0.05$ - but a statistically more significant improvement in the results was achieved after 8-fold performance of the exercise), after 2 , and then after 5 repeats for exercise 3 (Scheffe post-hoc test, $p<0.05$ ), after 5 repetitions of exercise 4 (post-hoc Scheffe test, $p<0.05$ ), after two repetitions of exercise 5 (Scheffe post-hoc test, $p<0.05)$, and at 2 , and then 3 repetitions for exercise 6 (post-hoc Scheffe test, $p<0.05$ ).

In the classical group, a statistically significant improvement of the average results was obtained: for exercise 1 after 2 , and then 8 repeats (post-hoc Scheffe test, $p<0.05)$ for exercise 2 after 2 repeats (Scheffe post-hoc test, $p<0.05$ ), for exercise 3 after

Table I. Demographic data

\begin{tabular}{|c|c|c|c|c|}
\hline Parameter & \multicolumn{2}{|c|}{$2009 / 2010$} & \multicolumn{2}{|c|}{$2010 / 2011$} \\
\hline \multicolumn{5}{|c|}{ Laparoscopic skills training (LapSkills) } \\
\hline Number of students & \multicolumn{2}{|l|}{35} & \multicolumn{2}{|l|}{27} \\
\hline Female & 14 & $40 \%$ & 13 & $48 \%$ \\
\hline Male & 21 & $60 \%$ & 14 & $52 \%$ \\
\hline Mean of age [year] & \multicolumn{2}{|c|}{$23.6(20-34)$} & \multicolumn{2}{|c|}{$23.7(20-31)$} \\
\hline \multicolumn{5}{|c|}{ Classic skills training (ClassSkills) } \\
\hline Number of students & \multicolumn{2}{|c|}{44} & \multicolumn{2}{|l|}{54} \\
\hline Female & 18 & $40 \%$ & 29 & $53 \%$ \\
\hline Male & 26 & $60 \%$ & 25 & $47 \%$ \\
\hline Mean of age [year] & \multicolumn{2}{|c|}{$23.4(20-30)$} & \multicolumn{2}{|c|}{$23.5(20-48)$} \\
\hline
\end{tabular}


2 repeats (Scheffe post-hoc test, $p<0.05$ ), for exercise 4 after 2 and then after 6 and 7 repeats (post-hoc Scheffe test, $p<0.05)$, for exercise 5 after 3 repetitions (Scheffe post-hoc test, $p<0.05$ ), similar to exercise 6 after 3 repetitions (Scheffe post-hoc test, $p<0.05)$.

Stabilization of the results (plateau) was obtained: a) in the laparoscopic group: for exercise 1 after 2 repetitions (Scheffe post-hoc, $p>0.7$ ), for exercise 2 after 5 repetitions (Scheffe post-hoc test, $p>0.7$ ), for exercise 3 after 5 repetitions (Scheffe post-hoc test, $p>0.7$ ), for exercise 4 after 5 repetitions (Scheffe post-hoc test, $p>0.7$ ), for exercise 5 after 2 repetitions (Scheffe post-hoc test, $p>0.7$ ), and for exercise 6 after 3 repetitions (Scheffe post-hoc test $p>0.7$ ), b) for the classical group: for exercise 1 after 2 repetitions a temporary plateau, which then was reduced after
8 repetitions (Scheffe post-hoc test, $p>0.7$ ), for exercise 2 after 2 repeats (Scheffe post-hoc test, $p>0.7$ ), for exercise 3 after 2 repeats (Scheffe post-hoc test, $p>0.7$ ), for exercise 4 a plateau was not achieved constant improvement of the results was observed until the end of the training ( 7 repetition), for exercises 5 after 3 repetitions (Scheffe post-hoc test, $p>0.7$ ) and for exercise 6 after 3 repeats (Scheffe post-hoc test, $p>0.7)$.

Based on stable measurements of exercise performance (plateau) there were estimated standards for each practice consisting of the mean + 1 SD (Table II and III). The standard was based on the results of 30 students participating in the laparoscopic training and 30 in classical training in previous years. Persons forming the control group were excluded from further analysis. Currently, the authors are carrying out

Table II. Time limits for tasks and percentage of students who performed task over the time limit - laparoscopic skills training group

\begin{tabular}{|lcccccc|}
\hline Number of task & 1 & 2 & 3 & 4 & 5 & 6 \\
\hline Medium time of performing a task (at the end of training) [s] & 71.5 & 239.5 & 293.2 & 215.2 & 196.8 & 195.3 \\
\hline Standard deviation (at the end of training) [s] & 30.3 & 248.2 & 222.5 & 144.6 & 139.9 & 142.9 \\
\hline Time limit for a task (medium time of control group + SD) [s] & 101.8 & 487.8 & 515.7 & 359.8 & 336.7 & 338.3 \\
\hline Number of students over time limit in first attempt & 48 & 41 & 38 & 31 & 25 & 15 \\
\hline Percentage of students over time limit in first attempt [\%] & 74 & 63 & 58 & 48 & 38 & 56 \\
\hline Number of students over time limit in last attempt & 4 & 6 & 5 & 9 & 11 & 3 \\
\hline Percentage of students over time limit in last attempt [\%] & 6 & 9 & 8 & 14 & 17 & 5 \\
\hline Percentage of students who improved time score [\%] & 92 & 85 & 87 & 71 & 56 & 80 \\
\hline
\end{tabular}

Table III. Time limits for tasks and percentage of students who performed task over the time limit - classic skills training group

\begin{tabular}{|lcccccc|}
\hline Number of task & 1 & 2 & 3 & 4 & 5 & 6 \\
\hline Medium time of performing a task (at the end of training) [s] & 613.0 & 116.0 & 473.1 & 201.1 & 286.3 & 155.3 \\
\hline Standard deviation (at the end of training) [s] & 174.9 & 50.2 & 393.9 & 102.2 & 188.7 & 88.5 \\
\hline Time limit for a task (medium time of control group + SD) [s] & 788.0 & 166.2 & 867.1 & 303.4 & 475.1 & 243.8 \\
\hline Number of students over time limit in first attempt & 84 & 55 & 73 & 47 & 32 & 25 \\
\hline Percentage of students over time limit in first attempt [\%] & 86 & 56 & 74 & 48 & 33 & 26 \\
\hline Number of students over time limit in last attempt & 13 & 16 & 15 & 11 & 9 & 2 \\
\hline Percentage of students over time limit in last attempt [\%] & 13 & 16 & 15 & 11 & 9 & 2 \\
\hline Percentage of students who improved time score [\%] & 85 & 71 & 79 & 77 & 72 & 92 \\
\hline
\end{tabular}


work on setting standards appropriate for postgraduate trainees and general surgery residents.

Doing three repetitions of the standard practice was considered as the result pointer to new qualification. After completing the training programme, $78.9 \%$ of participants achieved results in the normal range, including $78.5 \%$ in the laparoscopic arm and $79.3 \%$ in the classic arm. The highest percentage of the results was achieved in the normal range for exercise 1 in the laparoscopic arm and 6 in the classic arm (92\%), and the lowest for exercise 5 in the laparoscopic arm (56\%). Detailed percentages of participants meeting the requirements of specific exercises at the end of the programme are presented in Table II and III.

After the analysis of surveys conducted after completion of the course, it was found that:

a) $84 \%$ of participants considered the structured form of training very valuable;

b) $78.5 \%$ considered the introduction of competition components valuable, motivating and reasonable;

c) $81 \%$ pledged themselves to take part in such organized training again, if such a possibility arises.

\section{Discussion}

In this work, we have presented for the first time in the Polish literature, and as one of the few instances in world literature, a fully structured and operationalized training programme in basic manual skills in classical and laparoscopic surgery. Our proposed educational programme was effective, as indicated by the high interest in the implementation of training standards (78.5\% for the laparoscopic arm and $79.3 \%$ for the classic arm), as well as enjoying respect among students, as demonstrated by the results of questionnaires $(84 \%$ considered it a valuable programme, and $81 \%$ would like to participate in its subsequent editions). It should be noted that only a group of students who chose this programme themselves was analysed, so it could be assumed that these rates would be lower in the group of students not interested in surgery. Participants emphasized the need to introduce more such training programmes in other areas of medicine. Similar opinions can be found in Polish and world literature [9-13], which also highlights the long-term financial benefits resulting from the implementation of similar training programmes $[12,13]$. Specialized training programmes, introduced as elective classes during pre-diplo- ma training, though sometimes considered as too expensive, are making it easier for students to decide their future medical career. Before the training 54\% of the students wanted to pursue a career in surgery, the percentage after the training remained constant, while the number of students hesitating decreased from $24 \%$ to $10 \%$ but increased from $22 \%$ to $37 \%$ in those who chose non-surgery areas. The examined group of students - volunteers after completing training - clearly polarized on choosing a surgery or non-surgery career, but this tend should be compared with the general population of medical students, which requires further examination. Finally, as shown by other authors, laboratory training improves safety in the operating room [14], and improves the performance during the first surgery operations [15-19].

The presented educational programme is a complete teaching model, without the need to possess a virtual training simulator which was described in the methodological part of this work. Virtual trainer was primarily a measurement tool but was not determinating the effectiveness of training [20]. A similar approach was presented by other authors, who recognized the measuring value of virtual trainers, but they questioned the practical advantages due to the still insufficient scope of reflecting reality in virtual circumstances [21]. On the other hand, it should be noted that the virtual training software is subject to constant modifications. The range of available software for performing surgery practice is expanding as well.

Based on the study, the normative range for each exercise was estimated, allowing for further motivation of the students, and also allowing one to identify participants who will require more work in order to achieve satisfactory results. A plateau was achieved already after 2 or 3 repetitions in half exercise, which indicates that, in accordance to those exercises, the programme described by us could be considered as even too long. On the other hand, obtaining good results in more complex exercises (4, 5 and 6 for both training arms) could be related to the acquisition of substantial skill in performing simpler exercises, which was also an incentive. Moreover, in some of the exercises a double plateau effect was achieved, and in one of them the improvement in the results was observed until the completion of the course, making it impossible to achieve a plateau. Thus, it remains unsolved whether the result would have been comparable to the reduced stability long-term 
results. This aspect of the research, including the assessment of long-term results in the form of the stability of the results at 12 months after the end of the programme, is subject to further research by our team.

In addition, it should be noted that there is still imperfect assessment methodology in the classic arm. In this area the introduction of video coaching (analysis of video recordings of exercises) should be considered. This methodology is a proven teaching technique used in sports and air training [22-28]. Its use is also an alternative assessment in the laparoscopic arm in centres that do not have virtual trainers.

\section{Conclusions}

Manual skills training for surgery should be carried out on the basis of a structured programme similar to that proposed by our team. Training with use of modern teaching aids is an important addition to surgical training. This form of exercise allows one to achieve good and stable results, as well as the high satisfaction of trainees.

\section{References}

1. Peters JH, Fried GM, Swanstrom LL, et al.; SAGES FLS Committee. Development and validation of a comprehensive program of education and assessment of the basic fundamentals of laparoscopic surgery. Surgery 2004; 135: 21-7.

2. Ritter EM, Scott DJ. Design of a proficiency-based skills training curriculum for the fundamentals of laparoscopic surgery. Surg Innov 2007; 14: 107.

3. Edelman DA, Mattos MA, Bouwman DL. FLS skill retention (learning) in first year surgery residents. J Surg Res 2010; 163: 24-8.

4. Mashaud LB, Castellvi AO, Hollett LA, et al. Two-year skill retention and certification exam performance after fundamentals of laparoscopic skills training and proficiency maintenance. Surgery 2010; 148: 194-201.

5. Castellvi AO, Hollett LA, Minhajuddin A, et al. Maintaining proficiency after fundamentals of laparoscopic surgery training: a 1-year analysis of skill retention for surgery residents. Surgery 2009; 146: 387-93.

6. Scott DJ, Ritter EM, Tesfay ST, et al. Certification pass rate of $100 \%$ for fundamentals of laparoscopic surgery skills after proficiency-based training. Surg Endosc 2008; 22: 1887.

7. Buzink S, Soltes M, Radonak J, et al. Laparoscopic Surgical Skills programme: preliminary evaluation of grade I level 1 courses by trainees. Videosurgery Miniinv 2012; 7: 188-92.

8. van Dongen KW, Ahlberg G, Bonavina L, et al. European consensus on a competency-based virtual reality training program for basic endoscopic surgical psychomotor skills. Surg Endosc 2011; 25: 166-71.
9. Stefanidis D, Heniford BT. The formula for a successful laparoscopic skills curriculum. Arch Surg 2009; 144: 77-82.

10. Gruca Z, Kobiela J, Stefaniak T, et al. Surgical simulators in didactics of minimally invasive techniques. Videosurgery Miniinv 2008; 3: 30-4.

11. Budziński R, Michalik M, Frask A, et al. Education in laparoscopic surgery. Videosurgery Miniinv 2008; 3: 22-9.

12. Berg DA, Milner RE, Fisher CA, et al. A cost-effective approach to establishing a surgical skills laboratory. Surgery 2007; 142: 712-21.

13. Harrington DT, Roye GD, Ryder BA, et al. A time-cost analysis of teaching a laparoscopic entero-enterostomy. J Surg Educ 2007; 64: 342-5.

14. Ahlberg G, Enochsson L, Gallagher AG, et al. Proficiency-based virtual reality training significantly reduces the error rate for residents during their first 10 laparoscopic cholecystectomies. Am J Surg 2007; 193: 797-804.

15. Hyltander A, Liljegren E, Rhodin PH, et al. The transfer of basic skills learned in a laparoscopic simulator to the operating room. Surg Endosc 2002; 9: 1324-8.

16. Kundhal PS, Grantcharov TP. Psychomotor performance measured in a virtual environment correlates with technical skills in the operating room. Surg Endosc 2009; 3: 645-9.

17. Larsen CR, Soerensen JL, Grantcharov TP, et al. Effect of virtual reality training on laparoscopic surgery: randomised controlled trial. Surg Endosc 2011; 25: 166-71.

18. Schijven MP, Jakimowicz JJ, Broeders IA, et al. The Eindhoven laparoscopic cholecystectomy training course improving operating room performance using virtual reality training: results from the first E.A.E.S. accredited virtual reality trainings curriculum. Surg Endosc 2005; 9: 1220-6.

19. Seymour NE, Gallagher AG, Roman SA, et al. Virtual reality training improves operating room performance: results of a randomized, double-blinded study. Ann Surg 2002; 4: 458-63.

20. Łaski D, Stefaniak TJ, Makarewicz W, et al. Structuralized boxtrainer laparoscopic training significantly improves performance in complex virtual reality laparoscopic tasks. Videosurgery Miniinv 2012; 7: 27-32.

21. Jowett N, LeBlanc V, Xeroulis G, et al. Surgical skill acquisition with self-directed practice using computer-based video training. Am J Surg 2007; 193: 237-42.

22. Larsen CR, Oestergaard J, Ottesen BS, Soerensen JL. The efficacy of virtual reality simulation training in laparoscopy: a systematic review of randomized trials. Acta Obstet Gynecol Scand 2012; 91 : 1015-28.

23. Schmitt PJ, Agarwal N, Prestigiacomo CJ. From planes to brains: parallels between military development of virtual reality environments and virtual neurological surgery. World Neurosurg 2012; 78: 214-9.

24. Gillespie B, Xiao J, Perkins N, et al. The instrumented instrument: characterization and training of manual skill in open suturing. Stud Health Technol Inform 2008; 132: 141-6.

25. Jamshidi R, LaMasters T, Eisenberg D, et al. Video self-assessment augments development of videoscopic suturing skill. J Am Coll Surg 2009; 209: 622-5. 
26. Beard JD. Education and Training Committee of the Vascular Society of Great Britain and Ireland. Setting standards for the assessment of operative competence. Eur J Vasc Endovasc Surg 2005; 30: 215-8.

27. Driscoll PJ, Paisley AM, Paterson-Brown S. Video assessment of basic surgical trainees' operative skills. Am J Surg 2008; 196: 265-72.

28. Gambadauro P, Magos A. Digital video recordings for training, assessment, and revalidation of surgical skills. Surg Technol Int 2010; 20: 36-9

Received: 6.07.2012, accepted: 29.12.2012. 